\title{
Research on Line of Sight Angle Rate Reconstruction based on Strap down Seeker
}

\author{
Bo $\mathrm{Ji}^{\mathrm{a}}$, Cong Nie ${ }^{\mathrm{b}}, \mathrm{Ke}_{\text {Zhang }}{ }^{\mathrm{c}}$ \\ School of Astronautics, Northwestern Polytechnic University, Xi'an 710072 China \\ ajibonwpu@163.com, ${ }^{\mathrm{b}}$ niecong@163.com, ${ }^{\mathrm{c}}$ zhangke@nwpu.edu.cn
}

\begin{abstract}
Keywords: Strap down Seeker, LOS Rate Reconstruction, Central Difference Kalman filter, Digital Fading-Memory Filter.
\end{abstract}

\begin{abstract}
The detecting information of strap down seeker can't be applied to proportional navigation directly because it is coupled with missile attitude. So the method of line of sight (LOS) rate reconstruction for strap down seeker is proposed. The reconstruction result would be reflected by background noise and any inaccurate metrical information. Thus a LOS rate reconstruction method based on central difference approximation and Central Difference Kalman Filter (CDKF) was proposed to obtain more precise guidance information. Monte Carlo simulations confirmed the validity of the designed method.
\end{abstract}

\section{Introduction}

Homing missiles use a space stabilized seeker antenna in order to acquire and track the target. In conventional implementations of proportional navigation guidance systems, the degree to which the seeker is stabilized places fundamental limitations on the homing accuracy of the missile ${ }^{[1]}$. Strap down guidance system has become one of development trends of missile guidance system for its potential advantages of improving the system reliability and reducing cost. However, it also takes some fatal disadvantages. Firstly, only angles relative to body coordinate system can be measured by the strap down seeker. It leads to a high nonlinearity for guidance system. Secondly, more serious measurement noise was introduced in the system because of wider instantaneous field of view. It is difficult to extract inertial LOS rate, which is essential to the design of the guidance law ${ }^{[2]}$.

For these problems, Ying Liu proposed using filter method ${ }^{[3]}$ to obtain the target line of sight angle rate. Tinting Sun establish the mathematical model of strap down optical seeker ${ }^{[4]}$. Guiyang Zhang establish the second-order of the LOS angular based on the target maneuvering model ${ }^{[5]}$. Pei Wang proposed using Nonlinear Tracking-Differentiator to estimate the angle rate ${ }^{[6]}$.

Kalman Filter can obtain optimal unbiased estimation for linear system corrupted by white noise. Nevertheless, it cannot be applied to nonlinear system. The EKF expends dynamic model as a Taylor series about the current state estimate, and measurement model about the state prediction, and then linearization is achieved by neglecting second and higher order terms. But truncations decrease estimation precision; Calculation of Jacobian matrix's are nontrivial in most applications and usually leads to significant implemental difficulty. A new method, $\mathrm{CDKF}^{[7-9]}$ without and approximation for the nonlinear system model was proposed. The CDKF can predict the mean and covariance up to, even higher than the second order precision for Gauss distributed random variable.

In this paper, we focus on the strap down seeker which can only get LOS in body coordinates. The LOS first-order dynamic model was constructed. A LOS reconstruction filter was designed by using the model via the CDKF method. The results of simulation in MATLAB environment have showed that the method has a good performance on estimating the LOS angle. Meanwhile, the precision of the estimation can be improved. 


\section{The LOS Reconstruction Method}

We take the ground coordinate as the inertial frame. The definition and transformation of coordinates used in this paper are described as follows. The readers can refer to References ${ }^{[2]}$.

Inertial coordinates $O x_{I} y_{I} z_{I}$

Body coordinates $O x_{B} y_{B} z_{B}$

Inertial LOS coordinates $O x_{L} y_{L} z_{L}$

Body LOS coordinates $O x_{C} y_{C} z_{C}$

The yaw angle $q_{I H}$ and the pitch angle $q_{I V}$ in the inertial reference frame $S_{I}$ are essential to implement guidance law design. Since the optical system was fixed on the missile's body, only the yaw angle $q_{B H}$ and pitch angle $q_{B V}$ of LOS in body frame $S_{B}$ can be obtained. It is essential to decouple the missile attitudes information from the detecting information because of the missile attitudes are in flux.

The vector of target in inertial LOS coordinates and body LOS coordinates are all $\left(R_{r}, 0,0\right)$ ? According to the transformation matrices among the coordinates, we get the following equation:

$\left[\begin{array}{lll}x & y & z\end{array}\right]^{T}=T(\psi, \vartheta, \gamma) \cdot T\left(q_{B H}, q_{B V}\right) \cdot\left[\begin{array}{lll}R_{r} & 0 & 0\end{array}\right]^{T}=T\left(q_{I H}, q_{I V}\right) \cdot\left[\begin{array}{lll}R_{r} & 0 & 0\end{array}\right]^{T}$

The $q_{I H}$ and $q_{I V}$ can be obtained by the following equation

$$
\left[\begin{array}{ll}
q_{I H} & q_{I V}
\end{array}\right]^{T}=\left[\begin{array}{lll}
-\arctan \left(a_{3} / a_{1}\right) & \arcsin \left(a_{2}\right)
\end{array}\right]^{T}
$$

Where,

$$
\left\{\begin{aligned}
a_{1}= & \cos \vartheta \cos \psi \cos q_{B V} \cos q_{B H}-\sin \vartheta \cos \psi \cos \gamma \sin q_{B V}+\sin \psi \sin \gamma \sin q_{B V} \\
& -\sin \vartheta \cos \psi \sin \gamma \cos q_{B V} \sin q_{B H}-\sin \psi \cos \gamma \cos q_{B V} \sin q_{B H} \\
a_{2}= & \sin \vartheta \cos q_{B V} \cos q_{B H}+\cos \vartheta \cos \gamma \sin q_{B V}+\cos \vartheta \sin \gamma \cos q_{B V} \sin q_{B H} \\
a_{3}= & -\cos \vartheta \sin \psi \cos q_{B V} \cos q_{B H}+\sin \vartheta \sin \psi \cos \gamma \sin q_{B V}+\cos \psi \sin \gamma \sin q_{B V} \\
& +\sin \vartheta \sin \psi \sin \gamma \cos q_{B V} \sin q_{B H}-\cos \psi \cos \gamma \cos q_{B V} \sin q_{B H}
\end{aligned}\right.
$$

Thus, the decoupling equation was established. We can reconstruct the $q_{I H}$ and $q_{I V}$ according to missile attitudes which are measured by strapdown seeker. The inertial LOS rate can be obtained by differential network. To reduce the LOS angular rate's lag, another method was proposed as follows

$$
\left[\begin{array}{ll}
\dot{q}_{I H} & \dot{q}_{I V}
\end{array}\right]^{T}=\left[\begin{array}{ccc}
-\tan q_{I V} \cos q_{I H} & 1 & \tan q_{I V} \sin q_{I H} \\
\sin q_{I H} & 0 & \cos q_{I H}
\end{array}\right]\left[\begin{array}{llll}
\dot{q}_{I V} \sin q_{I H} & \dot{q}_{I H} & \dot{q}_{I V} \cos q_{I H}
\end{array}\right]^{T}
$$

The observation equation can be written as

$$
\left[\begin{array}{ll}
q_{B H} & q_{B V}
\end{array}\right]^{T}=\left[-\arctan \left(b_{3} / b_{1}\right) \arcsin \left(b_{2}\right)\right]^{T}
$$

Where, the definitions of $b_{1}, b_{2}$ and $b_{3}$ are similar to the form of $a_{1}, a_{2}$ and $a_{3}$.

\section{LOS rate estimation method based on Central Difference and CDKF}

The background noise of strapdown seeker and high nonlinearity of decoupling information make it difficult to calibrate the system noise. A simple method is that we defined the $\boldsymbol{\Omega}$ as $\boldsymbol{\Omega}_{c \text { Im }}^{I}$ plus Gaussian white noise. The foundation of CDKF is central difference approximation, which is a method for calculating the statistics of random variable via a nonlinear function ${ }^{[7-8]}$. We suppose that states are Gaussian random variables, therefore, only mean and covariance are essential to be approximated.

According to calibrations of seeker and navigation system, the statistic of BLOS noise vector $\mathbf{n}_{q}$, the BLOS rate noise vector $\mathbf{n}_{\omega q}$, the missile attitude noise vector $\mathbf{n}_{z}$, the rate gyro output noise vector $\mathbf{n}_{r g}$. The statistic of $\boldsymbol{\Omega}$ is calculated by central difference (CD) approximation. By using CD method, the mean and the covariance of output variables can be captured precisely up to the second order, and higher order information can be partially incorporated in the mean and the covariance, which leads to even higher accuracy. The implementation is extremely rapid because it is 
unnecessary to evaluate the Jacobians ${ }^{[12-14]}$. The mean $\boldsymbol{\Omega}_{\text {CIm }}^{I}$ and covariance $\mathbf{R}_{\mathrm{v}}$, obtained by using CD, are used as input and the covariance of zero-mean process noise $v$ respectively.

Ignored the high-order information of LOS angle, the Eq. 4 can be rewritten in discrete-time form as follows

$$
\left[\begin{array}{c}
q_{I H}(k+1) \\
q_{I V}(k+1)
\end{array}\right]=F\left\{q_{I H}(k), q_{I V}(k), \boldsymbol{\Omega}_{C I \mathrm{~m}}^{I}(k), v(k)\right\}=\left[\begin{array}{c}
q_{I H}(k) \\
q_{I V}(k)
\end{array}\right]+T \square M_{q}(k)\left\lfloor\left\{\mathbf{\Omega}_{C I \mathrm{~m}}^{I}(k)+v(k)\right\}\right.
$$

Where, $T$ is the strap down seeker's sampling time? It is the same that Eq. 5 can be rewritten in discrete form as follows

$$
\left[\begin{array}{c}
q_{B H}(k) \\
q_{B V}(k)
\end{array}\right]=H\left\{q_{I H}(k), q_{I V}(k), \psi(k), \vartheta(k), \gamma(k), \mathbf{n}_{z}(k), \mathbf{n}_{q}(k)\right\}=\left[\begin{array}{c}
-\arctan \left\{b_{3}(k) / b_{1}(k)\right\} \\
\arcsin \left\{b_{2}(k)\right\}
\end{array}\right]+\mathbf{n}_{q}(k)
$$

The details of LOS reconstruction filter by using CDKF algorithm can be described as follows Initialization

Let adjust parameter $h=\sqrt{3}$. Considering that the process noise $v_{k}$ and noise $n_{z}$ are not additive noises, and assumes that the process noise is not related with state in the initial estimate. The augmented state vector is selected as

$\mathbf{x}_{k}^{a}=\left[\begin{array}{ll}\left(\mathbf{x}_{k}^{x}\right)^{T} & \left(\mathbf{x}_{k}^{v}\right)^{T}\end{array}\right]^{T}=\left[\begin{array}{ll}\left(\mathbf{x}_{k}\right)^{T} & \left(v_{k}\right)^{T}\end{array}\right]^{T}$

The initial mean and covariance

$$
\hat{x}_{0}^{a}=\left[\hat{q}_{I H}(0) \quad \hat{q}_{I V}(0) \quad 0 \quad 0 \quad 0\right]^{T}, \mathbf{P}_{0}^{a}=\operatorname{diag}\left\{\mathbf{P}_{\mathbf{x} 0}, \mathbf{R}_{\mathbf{v}}\right\}
$$

Calculate the initial input $\boldsymbol{\Omega}_{c \mathrm{Im}}^{I}(0)$ and covariance of input noise $R_{v}(0)$

For sampling time $k=1,2, \cdots, \infty$, calculate the sigma points

$$
\boldsymbol{\chi}_{k-1}^{a}=\left[\begin{array}{lll}
\hat{\mathbf{x}}_{k-1}^{a} & \hat{\mathbf{x}}_{k-1}^{a}+h \sqrt{\mathbf{P}_{k-1}^{a}} & \hat{\mathbf{x}}_{k-1}^{a}-h \sqrt{\mathbf{P}_{k-1}^{a}}
\end{array}\right]
$$

Time updates

$$
\begin{gathered}
\boldsymbol{\chi}_{k \mid k-1}^{x}=F\left(\chi_{k-1}^{x}, \mathbf{\Omega}_{C I m}^{I}(k-1), \chi_{k-1}^{v}\right), \hat{\mathbf{x}}_{k}^{-}=\sum_{i=0}^{2 L} \mathbf{w}_{i}^{(m)} \boldsymbol{\chi}_{i, k \mid k-1}^{x} \\
\mathbf{P}_{\mathbf{x}_{k}}^{-}=\sum_{i=1}^{L}\left[\mathbf{w}_{i}^{(c 1)}\left(\boldsymbol{\chi}_{i, k \mid k-1}^{x}-\boldsymbol{\chi}_{i+L, k \mid k-1}^{x}\right)\left(\boldsymbol{\chi}_{i, k \mid k-1}^{x}-\boldsymbol{\chi}_{i+L, k \mid k-1}^{x}\right)^{T}+\mathbf{w}_{i}^{(c 2)}\left(\boldsymbol{\chi}_{i, k \mid k-1}^{x}+\boldsymbol{\chi}_{i+L, k \mid k-1}^{x}-2 \chi_{0, k \mid k-1}^{x}\right) \times\left(\chi_{i, k \mid k-1}^{x}+\boldsymbol{\chi}_{i+L, k \mid k-1}^{x}-2 \boldsymbol{\chi}_{0, k \mid k-1}^{x}\right)^{T}\right]
\end{gathered}
$$

Where,

$$
\begin{cases}w_{0}^{(m)}=\left(h^{2}-L\right) / h^{2} & i=0 \\ w_{i}^{(m)}=\frac{1}{2 h^{2}}, w_{i}^{(c 1)}=\frac{1}{4 h^{2}}, w_{i}^{(c 2)}=\frac{h^{2}-1}{4 h^{4}} & i=1,2, \cdots, 2 L\end{cases}
$$

Measurement updates equations

In the process of measurement updates, let the system state and measurement noise merge into one augmented state vector.

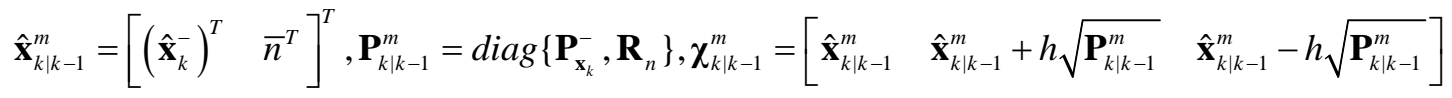

Thus, the measurement updates equations are

$$
\begin{gathered}
\boldsymbol{\gamma}_{k \mid k-1}=\mathbf{H}\left(\chi_{k \mid k-1}^{x}, \chi_{k \mid k-1}^{n}\right), \hat{\mathbf{y}}_{k}^{-}=\sum_{i=0}^{2 L} \mathbf{w}_{i}^{(m)} \boldsymbol{\gamma}_{i, k \mid k-1} \\
\mathbf{P}_{\hat{\mathbf{y}}_{k}}^{-}=\sum_{i=1}^{L}\left[\mathbf{w}_{i}^{(c 1)}\left(\boldsymbol{\gamma}_{i, k \mid k-1}-\boldsymbol{\gamma}_{i+L, k \mid k-1}\right)\left(\boldsymbol{\gamma}_{i, k \mid k-1}-\boldsymbol{\gamma}_{i+L, k \mid k-1}\right)^{T}\right. \\
\left.+\mathbf{w}_{i}^{(c 2)}\left(\boldsymbol{\gamma}_{i, k \mid k-1}+\boldsymbol{\gamma}_{i+L, k \mid k-1}-2 \boldsymbol{\gamma}_{0, k \mid k-1}\right) \times\left(\boldsymbol{\gamma}_{i, k \mid k-1}+\boldsymbol{\gamma}_{i+L, k \mid k-1}-2 \boldsymbol{\gamma}_{0, k \mid k-1}\right)^{T}\right] \\
\mathbf{P}_{\mathbf{x}_{k} \hat{y}_{k}}=\sqrt{w_{1}^{(c 1)} \mathbf{P}_{x_{k}}^{-}}\left[\boldsymbol{\gamma}_{1: L, k \mid k-1}-\boldsymbol{\gamma}_{L+1: 2 L, k \mid k-1}\right], \mathbf{K}_{k}=\mathbf{P}_{\mathbf{x}_{k} \hat{\hat{y}}_{k}} \mathbf{P}_{\hat{\mathbf{y}}_{k}}^{-1}, \hat{\mathbf{x}}_{k}=\hat{\mathbf{x}}_{k}^{-}+\mathbf{K}_{k}\left(\mathbf{y}_{k}-\hat{\mathbf{y}}_{k}^{-}\right), \mathbf{P}_{\mathbf{x}_{k}}=\mathbf{P}_{\mathbf{x}_{k}}^{-}-\mathbf{K}_{k} \mathbf{P}_{\hat{\mathbf{y}}} \mathbf{K}_{k}^{T}
\end{gathered}
$$

Calculation the input $\boldsymbol{\Omega}_{c \mathrm{Im}}^{I}(0)$ and covariance of input noise $R_{v}(k)$ in measurement values of seeker and navigation system by the method of CD.

Back to (2) 
Since the square root of the covariance matrix must be calculated when time updates, the method may has large computation. The square root of the covariance matrix can be calculated by Cholesky decomposition ${ }^{[9]}$. Compared with the standard form, the square root form not only improves the computational efficiency, but also to ensure the covariance matrix to be a positive definite matrix. Thus, more stable numerical calculation results can be obtained.

\section{Simulation Results}

In this section, the performance of LOS reconstruction filter is validated by a simple homing missile intercept simulation. The initial simulation condition are showed as follows.

Missile: $P_{m}=\left[\begin{array}{lll}0 & 7000 & 0\end{array}\right], V_{m}=306 \mathrm{~m} / \mathrm{s}, J_{x}=0.251 \mathrm{~kg} \cdot \mathrm{m}^{2}, J_{y}=J_{z}=6.606 \mathrm{~kg} \cdot \mathrm{m}^{2}$

Target: $P_{m}=\left[\begin{array}{lll}10000 & 0 & 1000\end{array}\right], V_{t}=\left[\begin{array}{lll}27 & 0 & 20\end{array}\right]$

Noise: $\quad \mathbf{n}_{q}=0.0001 \mathrm{rad}, \mathbf{n}_{z}=0.002 \mathrm{rad}, \mathbf{n}_{\omega q}=0.02 \mathrm{rad} / \mathrm{s}, \mathbf{n}_{\mathrm{rg}}=0.001 \mathrm{rad} / \mathrm{s}$

DFMF is used as low-pass filter and derivative network ${ }^{[10-11]}$, and its memory length parameter is 0.8 in simulations. The curves of simulation results are showed as Fig.1-4. Compared with the LOS calculated by solving directly and low-pass filter, good results are obtained by using LOS reconstruction filter, and then the accuracy of LOS estimation is improved by applying the output of the proposed filter. Fifty Monte Carlo simulations are performed. The mean and standard deviation of estimation error of LOS reconstruction angular rate by using EKF and CDKF are showed in Table 1. We can see that the proposed method has a more accurate precision to enhance the performance of guidance system compared with EKF.

Table 1. Mean of RMSE

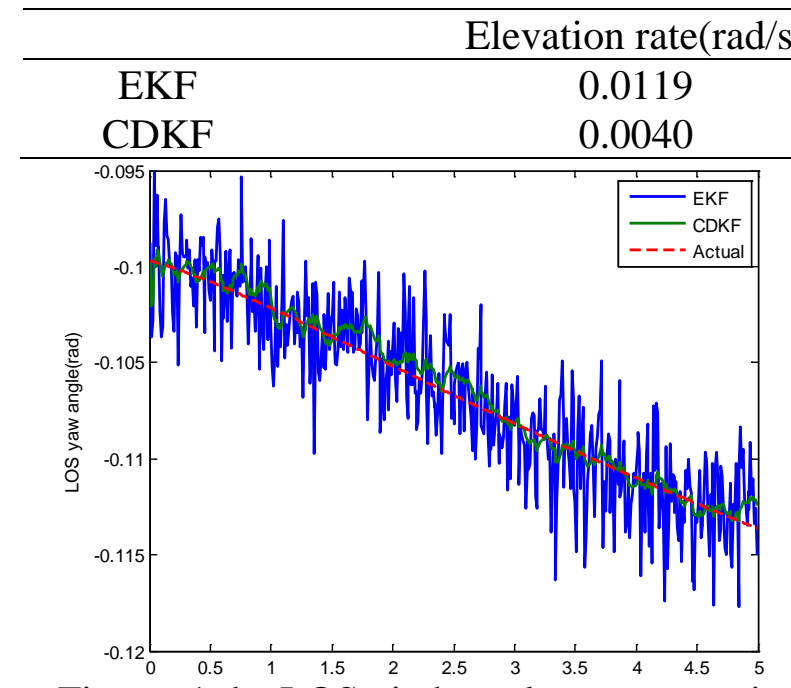

Figure. 1 the LOS pitch angle reconstruction

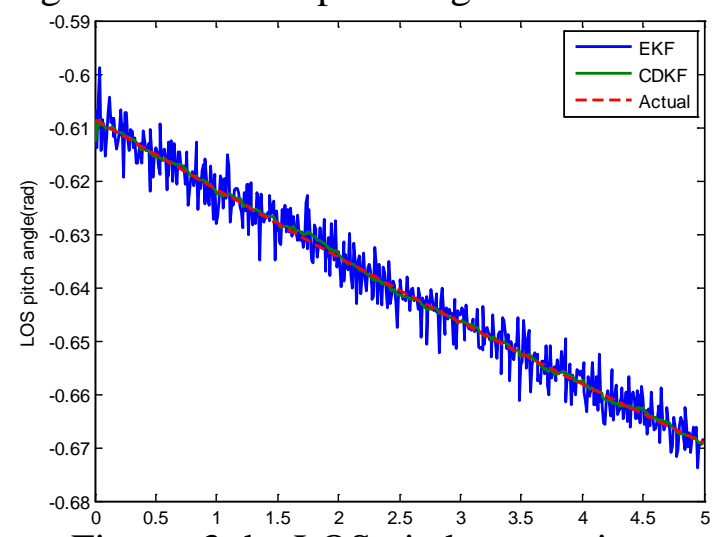

Figure. 3 the LOS pitch rate estimate

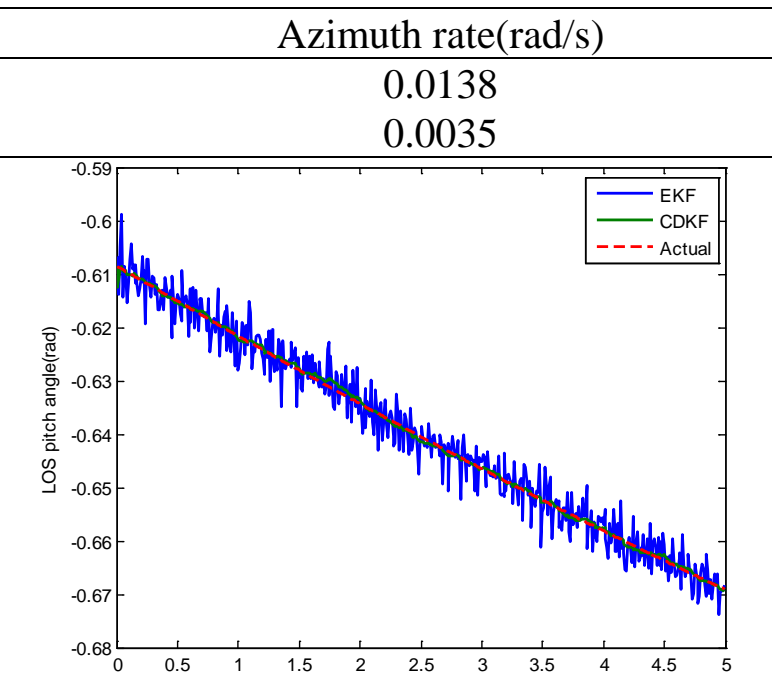

Figure. 2 the LOS yaw angle reconstruction

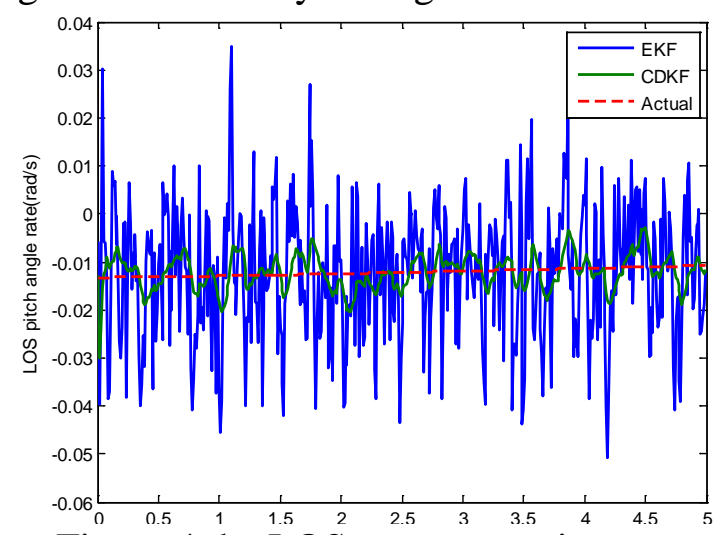

Figure.4 the LOS yaw rate estimate

\section{Conclusions}


By the reason of the high nonlinearity and serious measurement noise contained in the strap down imaging seeker guidance system, the CDKF is employed to estimate inertial LOS rate in this paper. Estimation results are compared with that of EKF by Monte Carlo simulations. These simulations show that the CDKF is superior to the EKF both in precision of estimation and velocity of convergence for the strap down imaging guidance system. Besides, calculation of Jacobian matrices is avoided for the CDKF, which is usually a complicated process, and may suffer singularity to make the EKF infeasible.

\section{References}

[1] Kim Dowan, Ryoo Chang-kyung, Kim Yongho, Kim Jongju. Guidance and control for missiles with a strapdown seeker[C]. //2011 11th International Conference on Control, Automation and Systems. Gyeonggi-do, Korea. 2011,p.969-972

[2] Ruiqing Zhou, Xinhua Liu, Shouxia Shi, Kaibin Wang. The Stable Tracking Technology of Strapdown Seeker[M]. Beijing: National Defence Industry Press, 2010

[3] Ying Liu, Weifeng Tian, Jiankang Zhao. Line of sight angle rate reconstruction for phased array strapdwon seeker [C] // 2013 International Conference on Intelligent System. Taiyuan. 2013, p. 196-201

[4] Tingting Sun, Hairong Chu, Hongguang Jia. Line of sight angular rate estimation of strapdown optical image seeker [J]. Chinese Optical Society. vol.34, June 2014.

[5] GuoJiang Zhang, Yu Yao, KeMao Ma. Line of Sight Rate Estimation of Strapdown Imaging Guidance System Based on Unscented Kalman Filter[C]// Proceedings of the Fourth International Conference on Machine Learing and Cybernetics. Guangzhou. 2005, p. 1574-1578

[6] Pei Wang,Ke Zhang, Cong Nie. Research on line of sight rate estimation of strapdown seeker[C]//2014 International Conference on Mechatronics Engineering and Computing Technology. United States:Trans Tech Publications. 2014, p.556-562

[7] Xue Liu, Shuhong Jiao, Liang Sun. Improved central difference Kalman Filter for Single Observer passive location[J]. Changchun: Journal of Jilin University. 2011.11, p.1777-1782

[8] Ping Han, Haoliang Gan, Weikun He. Aircraft Attitude Estimation Based on Central Difference Kalman Filter[C]//2012 11th International Conference on Signal Processing. Beijing,China: Institude of Electrical and Electronics Engineers Inc. 2012,p.294-298

[9] Jun Li, Huazhong Lu, Zhou Yang, Feng Pei. State-of-charge estimation and charge equalization for electric agricultural machinery using Square-Root Central Difference Kalman Filter[C]//2011 Internation Conference on Transportation,Mechanical, and Electrical Engineering. United States: IEEE Computer Society. 2011, p.966-969

[10] Xue Liu, Shuhong Jiao, Liang Sun. Improved central difference Kalman Filter for Single Observer passive location[J]. Changchun: Journal of Jilin University. 2011.11, p. 1777-1782

[11] Ping Han, Haoliang Gan, Weikun He. Aircraft Attitude Estimation Based on Central Difference Kalman Filter[C]//2012 11th International Conference on Signal Processing. Beijing,China: Institude of Electrical and Electronics Engineers Inc. 2012, p. 294-298

[12] Jun Li, Huazhong Lu, Zhou Yang, Feng Pei. State-of-charge estimation and charge equalization for electric agricultural machinery using Square-Root Central Difference Kalman Filter[C] // 2011 Internation Conference on Transportation, Mechanical, and Electrical Engineering. United States: IEEE Computer Society. 2011, p.966-969

[13] Hufei Zhu, Chen Wen, Bin Li, Feifei Gao. An Improved Square-Root Algorithm for V-BLAST Based on Efficient Inverse Cholesky Factorization. Wireless Communications[J]. Piscataway: IEEE Transcations on. 2011, p.43-48. 